\title{
Stability Analysis for Seed Cotton Yield and its Component Traits in Hybrids of Desi Cotton (Gossypium arboreum L.)
}

\author{
V.N. Chinchane ${ }^{1 *}$, D.B. Deosarkar ${ }^{2}$ and H.V. Kalpande ${ }^{3}$ \\ Department of Agricultural Botany, (Genetics and Plant Breeding), Vasantrao Naik \\ Marathawada Krishi Vidyapeeth, Parbhani-431402 (M.S.), India \\ *Corresponding author
}

\begin{tabular}{|l|}
\hline K e y w o r d s \\
Cotton, Stability, \\
Yield, Fibre \\
strength
\end{tabular}

\section{A B S T R A C T}

Cotton is a long duration crop and grown over a wide range of environments. It is, therefore necessary to evaluate cotton genotypes in different environments to know its stability in varying environmental conditions. The knowledge of kind and magnitude of $G$ $\mathrm{x} \mathrm{E}$ interaction has become essential to the plant breeder in taking the decisions concerning breeding methods, selection programmes and their testing procedures in crop plants. Stability in yield is a major consideration in breeding of rainfed cotton which is highly influenced by erratic and uncertain rainfall conditions. Development of stable genotypes with high yield potential under rainfed condition is need of the day to meet the growing domestic demands of medium and superior medium staple cotton. Fifty six crosses with fifteen parents and four checks viz., PKVDH1, PKV Suvarna, NACH 12 and PA 255 were grown in Randomized Block Design with two replications. The experiment was conducted under rainfed condition at three locations viz., Cotton Research Station, MB Farm, Parbhani $\left(\mathrm{L}_{1}\right)$, Cotton Research Station, Nanded $\left(\mathrm{L}_{2}\right)$, and Agricultural Research Station, Badnapur $\left(\mathrm{L}_{3}\right)$ during kharif, 2016.The observations are recorded days to $50 \%$ flowering, days to $50 \%$ boll bursting, plant height, no. of sympodia per plant, no. of bolls per plant, boll weight, seed cotton yield per plant, seed index, lint index, harvest index and days to maturity. This experiment has resulted in identification of four parents i.e. PA 809, PA 785, HD 514, and PA 832 which were found to be stable and responsive to the low yielding environment which can be used as parents for improving yield stability of the cotton genotypes. The results showed that the stable crosses for the seed cotton yield per plant were PAIG 346 x JLA 794, PAIG 346 x DWDa 1402, PA 785 x CNA 449, PAIG 346 x CNA 449, PAIG 346 x HD 514 and PA 801 x HD 514 which have also responded well to favourable environments.

\section{Introduction}

Genotype $\mathrm{x}$ environment interactions is of major importance to the plant breeder in developing improved genotypes. When genotypes are compared over a series of environments, the relative rankings usually differ. This causes difficulty in demonstrating the significant superiority of any genotype. This interaction is usually present whether the varieties are pure lines, single cross, double cross hybrids, top crosses, $\mathrm{S}_{1}$ lines or any other material with which the breeder may be working. 
Cotton plant has indeterminate growth habit which is greatly influenced by environmental conditions. Under high fertility conditions, cotton plant can attain more plant height, more nodes, more sympodia, delayed flowering whereas under stress conditions the plants may remain stunted with less monopods, sympodia and nodes or may initiate flowering very early.

Similarly the final yields of cotton, which is governed by polygenes and which are highly influenced by environment is a very complex character and have several components. To improve yield, in actual practice a breeder has to select individuals on the basis of phenotypic expression. Many characters of economic worth are quantitatively inherited and manifest high genotype environment effects.

Hence the present study was carried out to determine the stability of parents and crosses to indentify most stable genotypes for future breeding programme.

\section{Materials and Methods}

The present study comprised of seven females (lines) and eight males (testers) with four standard checks thus making $56 \quad \mathrm{~F}_{1} \mathrm{~s}$ using Line $\mathrm{x}$ Tester mating design.

These lines, testers and hybrids along with four checks were sown during kharif, 2016 at three locations viz., Cotton Research Station, MB Farm, Parbhani $\left(\mathrm{L}_{1}\right)$, Cotton Research Station, Nanded $\left(\mathrm{L}_{2}\right)$, and Agricultural Research Station, Badnapur $\left(\mathrm{L}_{3}\right)$.

The observations recorded on days to $50 \%$ flowering, days to $50 \%$ boll bursting, plant height $(\mathrm{cm})$, number of sympodia per plant, number of boll per plant, boll weight ( $\mathrm{g}$ ), seed cotton yield per plant $(\mathrm{g})$, seed index $(\mathrm{g})$, lint index $(\mathrm{g})$, harvest index $(\%)$. Analysis was carried out as per the method suggested by Eberhart and Russell (1968).

\section{Results and Discussion}

From the ANOVA it was evident that mean squares for genotypes $\mathrm{x}$ environment were significant for number of sympodia per plant, number of bolls per plant, boll weight and seed cotton yield per plant which indicated inconsistency of performance of cotton genotypes across the environments for these characters. The significance of environment linear component for all the characters indicated considerable differences among the environments and their predominant effects on the traits. The significance of genotypes $\mathrm{x}$ environment linear component for plant height, number of sympodia per plant, number of bolls per plant, boll weight, seed cotton yield per plant, seed index and days to maturity indicated preponderance of linear component in these traits and hence prediction appeared possible. The pooled deviation for most of characters except number of sympodia per plant and number of bolls per plant was non-significant indicated the performance of the genotypes for these traits could be predictable.

Among all the character, yield is a very complex character which is controlled by polygene thereby showing the continuous variation in interaction with environment. It is also one of the most important characters breeder aims at. Therefore it would be wise to identify stable crosses on the basis of yield followed by other characters as it is the highly prone to environmental differences. Out of fifteen parents, five parents showed highly stable performance across the environments. They exhibited high mean than parental mean and non-significant deviation from regression. Among parents line PA 785 was found to be highly stable for yield per plant followed by PA 809 and HD 514. Out of fifty six crosses, twenty two crosses were widely stable with high mean than hybrid mean and nonsignificant deviation from regression (Table $1)$. 
Table.1 Estimates of stability parameters of genotypes over three environments

\begin{tabular}{|c|c|c|c|c|c|c|c|c|c|c|c|c|c|}
\hline \multirow{2}{*}{$\begin{array}{l}\text { Sr. } \\
\text { No. }\end{array}$} & \multirow[t]{2}{*}{ Genotype } & \multicolumn{3}{|c|}{ Days to $50 \%$ flowering } & \multicolumn{3}{|c|}{ Days to $50 \%$ boll bursting } & \multicolumn{3}{|c|}{ Plant height (cm) } & \multicolumn{3}{|c|}{ Number of sympodia /plant } \\
\hline & & Mean & Bi & $S^{2} \mathrm{di}$ & Mean & $\mathbf{B i}$ & $S^{2} d i$ & Mean & Bi & $\mathbf{S}^{2} \mathrm{di}$ & Mean & Bi & $S^{2} d i$ \\
\hline & Lines & & & & & & & & & & & & \\
\hline 1 & PA 801 & 71.16 & 1.64 & 0.83 & 115.33 & $-0.69 *$ & -1.16 & 106.30 & 1.08 & 6.64 & 14.83 & 1.00 & -2.12 \\
\hline 2 & PA 740 & 73.50 & 1.10 & -0.49 & 118.66 & 0.48 & -0.58 & 104.96 & 1.14 & -8.58 & 13.50 & $0.20 *$ & -2.64 \\
\hline 3 & PA 812 & 71.00 & 1.10 & -0.49 & 116.33 & 0.24 & -1.02 & 99.53 & 1.00 & -8.31 & 15.50 & $0.16^{*}$ & -2.65 \\
\hline 4 & PA 809 & 72.16 & 1.12 & -0.33 & 117.00 & 1.34 & -0.28 & 104.70 & 1.23 & -8.12 & 15.77 & 0.09 & -0.15 \\
\hline 5 & PA 785 & 73.00 & 1.39 & -0.65 & 118.50 & 1.34 & -0.28 & 102.10 & $0.70 *$ & -9.37 & 16.43 & 0.46 & -2.22 \\
\hline 6 & PA 832 & 71.50 & 1.39 & -0.65 & 117.50 & 0.93 & -0.96 & 107.03 & 1.13 & -8.59 & 15.87 & 0.47 & -2.66 \\
\hline \multirow[t]{2}{*}{7} & PAIG 346 & 72.66 & -0.56 & -0.61 & 119.16 & -0.45 & -1.07 & 103.40 & 1.21 & -8.89 & 13.50 & 0.43 & -2.45 \\
\hline & Testers & & & & & & & & & & & & \\
\hline 1 & AKA 8 & 70.00 & 1.10 & -0.49 & 115.00 & -0.21 & -0.68 & 98.20 & 0.94 & -9.30 & 16.17 & 0.43 & -2.52 \\
\hline 2 & PhuleDhanwantary & 71.50 & 1.39 & -0.65 & 116.16 & 1.83 & -1.14 & 71.00 & $0.49 *$ & -9.36 & 12.23 & $0.17 *$ & -2.67 \\
\hline 3 & CNA 449 & 73.66 & 1.93 & -0.41 & 118.00 & 2.07 & -1.12 & 106.23 & 0.92 & -5.36 & 14.93 & -0.05 & -0.77 \\
\hline 4 & HD 514 & 71.00 & 1.10 & -0.49 & 115.83 & 0.24 & -1.02 & 87.70 & 0.56 & -9.14 & 13.80 & $0.34 *$ & -2.67 \\
\hline 5 & DWDa 1402 & 71.33 & $3.05^{*}$ & -0.70 & 117.00 & 2.07 & -1.12 & 111.20 & $0.61 *$ & -9.37 & 16.03 & $0.34^{*}$ & -2.68 \\
\hline 6 & JLA 794 & 72.66 & 1.12 & -0.33 & 117.66 & 1.83 & -1.14 & 113.70 & 0.27 & 13.49 & 11.83 & 0.25 & -1.93 \\
\hline 7 & Digvijay & 81.50 & 0.29 & -0.22 & 132.83 & 1.59 & -0.86 & 101.43 & 1.08 & -9.13 & 16.30 & 0.33 & -2.55 \\
\hline \multirow[t]{2}{*}{8} & G.Cot 23 & 80.00 & 1.39 & -0.65 & 131.33 & 1.79 & 0.41 & 99.23 & 0.35 & -9.02 & 15.43 & 0.41 & -0.97 \\
\hline & Crosses & & & & & & & & & & & & \\
\hline 1 & PA 801 x AKA 8 & 65.50 & 2.77 & -0.50 & 110.16 & 1.83 & -1.14 & 106.93 & 0.77 & -4.09 & 21.90 & 1.12 & -2.45 \\
\hline 2 & PA 801 x PhuleDhanwantary & 70.16 & -1.94 & -0.42 & 116.00 & 1.87 & -0.35 & 93.86 & 0.51 & -8.35 & 16.23 & 1.38 & -1.18 \\
\hline 3 & PA 801 x CNA 449 & 73.33 & 1.09 & 1.69 & 119.00 & 2.59 & 3.05 & 115.56 & 1.17 & -7.09 & 21.20 & 1.44 & -2.47 \\
\hline 4 & PA 801 x HD 514 & 70.33 & 1.66 & -0.68 & 115.00 & 2.07 & -1.12 & 117.96 & 1.24 & -8.70 & 23.33 & 1.19 & -2.38 \\
\hline 5 & PA 801 x DWDa 1402 & 69.33 & 2.23 & 0.80 & 113.83 & 1.79 & 0.41 & 120.90 & 1.57 & -6.65 & 21.60 & 1.17 & -2.43 \\
\hline 6 & PA 801 x JLA 794 & 72.33 & 1.37 & 0.02 & 117.50 & 1.87 & -0.35 & 122.60 & 1.53 & -6.83 & 21.63 & 0.60 & $11.09 *$ \\
\hline 7 & PA 801 x Digvijay & 79.16 & -1.66 & -0.68 & 125.16 & 2.76 & -1.09 & 111.16 & 1.61 & -7.91 & 15.97 & 1.93 & -2.00 \\
\hline 8 & PA 801 x G.Cot 23 & 78.16 & 0.01 & 0.46 & 124.50 & 0.73 & 0.15 & 110.86 & 1.53 & -9.40 & 16.83 & 0.82 & $24.77 * *$ \\
\hline 9 & PA $740 \times$ AKA 8 & 71.00 & 1.67 & 0.14 & 116.33 & 0.45 & -1.07 & 112.73 & 1.49 & -6.00 & 17.67 & 1.59 & -1.76 \\
\hline 10 & PA 740 x PhuleDhanwantary & 73.00 & 0.81 & 0.64 & 119.66 & 2.24 & 1.29 & 94.63 & 0.57 & -7.59 & 17.07 & 1.37 & -2.52 \\
\hline
\end{tabular}


Table.1 (Contd...)

\begin{tabular}{|c|c|c|c|c|c|c|c|c|c|c|c|c|c|}
\hline \multirow{2}{*}{$\begin{array}{l}\text { Sr. } \\
\text { No. }\end{array}$} & \multirow[t]{2}{*}{ Genotype } & \multicolumn{3}{|c|}{ Days to $50 \%$ flowering } & \multicolumn{3}{|c|}{ Days to $50 \%$ boll bursting } & \multicolumn{3}{|c|}{ Plant height (cm) } & \multicolumn{3}{|c|}{ Number of sympodia /plant } \\
\hline & & Mean & $\mathbf{B i}$ & $S^{2} \mathrm{di}$ & Mean & $\mathbf{B i}$ & $S^{2} \mathrm{di}$ & Mean & $\mathbf{B i}$ & $S^{2} d i$ & Mean & Bi & $S^{2} d i$ \\
\hline 11 & PA 740 x CNA 449 & 73.33 & 1.37 & 0.02 & 118.50 & 0.73 & 0.15 & 118.43 & 0.14 & 3.35 & 21.07 & 1.46 & -1.59 \\
\hline 12 & PA 740 x HD 514 & 70.00 & 1.67 & 0.14 & 114.66 & -0.97 & 1.18 & 100.61 & 1.32 & -5.84 & 21.23 & 0.47 & $10.58^{*}$ \\
\hline 13 & PA 740 x DWDa 1402 & 71.50 & 1.67 & 0.14 & 116.16 & 1.10 & 0.59 & 119.23 & 1.55 & -8.85 & 20.77 & 0.60 & $9.41 *$ \\
\hline 14 & PA 740 x JLA 794 & 72.00 & 1.39 & -0.65 & 116.83 & 1.38 & -1.15 & 121.23 & 1.47 & -9.15 & 16.73 & 0.56 & $13.08^{*}$ \\
\hline 15 & PA 740 x Digvijay & 73.50 & 0.29 & -0.22 & 118.16 & 1.83 & -1.14 & 114.26 & 1.60 & -8.40 & 22.30 & 1.94 & -2.43 \\
\hline 16 & PA 740 x G.Cot 23 & 74.00 & -0.53 & $2.73 *$ & 117.50 & -3.00 & -0.73 & 112.80 & 0.11 & -6.95 & 21.33 & 2.12 & -2.56 \\
\hline 17 & PA 812 x AKA 8 & 67.33 & -0.01 & 0.46 & 111.83 & -2.04 & -0.41 & 105.83 & 1.17 & -8.50 & 21.40 & $1.50^{*}$ & -2.68 \\
\hline 18 & PA 812 x PhuleDhanwantary & 69.33 & 0.84 & 0.30 & 114.33 & 0.65 & -0.15 & 90.00 & -0.32 & -6.32 & 15.83 & 1.19 & -2.38 \\
\hline 19 & PA 812 x CNA 449 & 70.50 & 1.67 & 0.14 & 115.50 & 1.34 & -0.28 & 112.33 & 0.87 & -4.00 & 21.13 & 0.79 & $15.52 * *$ \\
\hline 20 & PA 812 x HD 514 & 67.50 & $0.00 *$ & -0.70 & 112.00 & 2.80 & 0.67 & 96.16 & 0.07 & -9.26 & 24.17 & 1.14 & -2.20 \\
\hline 21 & PA 812 x DWDa 1402 & 71.33 & 0.56 & -0.61 & 116.50 & 1.14 & -1.11 & 113.10 & 0.81 & -8.39 & 18.30 & 1.40 & -2.01 \\
\hline 22 & PA 812 x JLA 794 & 71.33 & 2.23 & 0.80 & 116.66 & 2.24 & 1.29 & 116.10 & 1.57 & -6.26 & 20.10 & 0.87 & $23.77 * *$ \\
\hline 23 & PA 812 x Digvijay & 71.83 & 0.27 & -0.55 & 117.16 & 1.83 & -1.14 & 113.80 & -2.81 & -7.48 & 17.93 & 1.66 & -0.25 \\
\hline 24 & PA 812 x G.Cot 23 & 72.83 & -1.12 & -0.33 & 118.50 & -1.14 & -1.11 & 111.66 & 1.51 & -3.40 & 20.33 & 0.68 & $9.13^{*}$ \\
\hline 25 & PA 809 x AKA 8 & 70.16 & 2.22 & -0.68 & 113.16 & 4.83 & -0.93 & 109.80 & 0.71 & -1.37 & 27.17 & 0.43 & -0.57 \\
\hline 26 & PA 809 x PhuleDhanwantary & 72.00 & 2.77 & -0.50 & 116.83 & 2.73 & -0.52 & 96.06 & 1.02 & -8.34 & 16.93 & 1.75 & -1.20 \\
\hline 27 & PA 809 x CNA 449 & 73.33 & 1.94 & -0.42 & 119.00 & 2.28 & -0.93 & 124.46 & 1.31 & -2.49 & 24.23 & 1.42 & -1.93 \\
\hline 28 & PA 809 x HD 514 & 70.66 & -0.27 & -0.55 & 115.16 & -2.31 & -0.82 & 97.93 & 0.89 & -5.00 & 21.93 & 1.47 & -2.61 \\
\hline 29 & PA 809 x DWDa 1402 & 71.00 & 1.39 & -0.65 & 115.50 & 1.55 & 1.52 & 122.86 & 1.35 & 0.10 & 20.53 & 1.68 & -2.47 \\
\hline 30 & PA 809 x JLA 794 & 72.50 & 2.20 & 0.17 & 117.83 & 2.11 & 0.49 & 121.70 & 1.49 & -9.28 & 20.93 & 1.72 & -0.91 \\
\hline 31 & PA 809 x Digvijay & 74.16 & 3.03 & 0.32 & 119.33 & 3.25 & -0.08 & 112.20 & 1.32 & -8.64 & 17.90 & 1.11 & $17.13^{* *}$ \\
\hline 32 & PA 809 x G.Cot 23 & 73.83 & -0.54 & -0.11 & 120.66 & 4.83 & -0.93 & 115.13 & 1.14 & -8.33 & 20.07 & 2.37 & -0.90 \\
\hline 33 & PA 785 x AKA 8 & 69.33 & $3.05 *$ & -0.70 & 114.00 & -0.21 & -0.68 & 110.86 & 1.43 & -2.13 & 22.03 & 1.50 & -2.09 \\
\hline 34 & PA 785 x PhuleDhanwantary & 72.50 & 1.10 & -0.49 & 117.50 & 0.93 & -0.96 & 94.26 & 0.64 & -3.88 & 18.07 & 0.67 & $10.44^{*}$ \\
\hline 35 & PA 785 x CNA 449 & 73.33 & 2.47 & 1.04 & 118.66 & 2.56 & -0.22 & 113.13 & 1.28 & -7.48 & 25.23 & 1.34 & -2.33 \\
\hline 36 & PA 785 x HD 514 & 68.83 & 4.43 & -0.62 & 112.33 & $-0.69 *$ & -1.16 & 95.90 & 1.05 & -0.45 & 21.27 & 1.63 & -1.19 \\
\hline 37 & PA 785 x DWDa 1402 & 72.83 & 1.66 & -0.68 & 118.16 & 1.62 & -0.90 & 116.90 & 2.11 & -3.81 & 20.80 & 1.74 & -1.82 \\
\hline 38 & PA 785 x JLA 794 & 73.66 & -1.66 & -0.68 & 119.33 & -2.56 & -0.22 & 115.87 & 1.42 & -0.54 & 20.73 & 1.19 & $18.76 * *$ \\
\hline 39 & PA 785 x Digvijay & 73.00 & 1.39 & -0.65 & 118.33 & 1.59 & -0.86 & 111.50 & 1.11 & -5.00 & 19.40 & 0.98 & $19.79 * *$ \\
\hline 40 & PA 785 x G.Cot 23 & 73.33 & 1.66 & -0.68 & 119.50 & 0.93 & -0.96 & 107.93 & 1.34 & -9.31 & 18.10 & 1.65 & -1.07 \\
\hline
\end{tabular}


Table.1 (Contd...)

\begin{tabular}{|c|c|c|c|c|c|c|c|c|c|c|c|c|c|}
\hline \multirow{2}{*}{$\begin{array}{l}\text { Sr. } \\
\text { No. }\end{array}$} & \multirow[t]{2}{*}{ Genotype } & \multicolumn{3}{|c|}{ Days to $50 \%$ flowering } & \multicolumn{3}{|c|}{ Days to $50 \%$ boll bursting } & \multicolumn{3}{|c|}{ Plant height (cm) } & \multicolumn{3}{|c|}{ Number of sympodia /plant } \\
\hline & & Mean & $\mathbf{B i}$ & $S^{2} d i$ & Mean & $\mathbf{B i}$ & $S^{2}$ di & Mean & Bi & $S^{2}$ di & Mean & $\mathbf{B i}$ & $S^{2} \mathrm{di}$ \\
\hline 41 & PA $832 \times$ AKA 8 & 68.33 & -1.40 & 1.00 & 113.33 & -2.04 & -0.41 & 112.66 & 1.11 & -1.51 & 17.87 & 0.66 & $8.90 *$ \\
\hline 42 & PA 832 x PhuleDhanwantary & 71.83 & -1.40 & 1.00 & 116.66 & -0.45 & -1.07 & 94.66 & 0.32 & -9.26 & 16.73 & 0.73 & $7.76^{*}$ \\
\hline 43 & PA 832 x CNA 449 & 72.00 & 2.49 & -0.65 & 117.00 & 1.87 & -0.35 & 118.40 & 1.20 & -8.98 & 24.50 & 1.44 & -1.52 \\
\hline 44 & PA 832 x HD 514 & 66.83 & 0.27 & -0.55 & 112.16 & $3.90 *$ & -1.17 & 100.40 & 1.12 & -8.92 & 16.13 & 1.21 & -2.22 \\
\hline 45 & PA 832 x DWDa 1402 & 70.83 & 0.84 & 0.30 & 115.50 & 0.41 & 0.77 & 118.26 & 0.93 & -8.08 & 18.20 & 1.31 & -1.33 \\
\hline 46 & PA 832 x JLA 794 & 72.33 & 1.37 & 0.02 & 117.50 & 0.73 & 0.15 & 119.03 & 1.80 & -7.05 & 18.33 & 1.36 & -2.45 \\
\hline 47 & PA 832 x Digvijay & 73.16 & -2.76 & -0.31 & 119.66 & -3.77 & $7.18 * *$ & 114.70 & 1.12 & -8.33 & 16.57 & 1.51 & 0.13 \\
\hline 48 & PA 832 x G.Cot 23 & 74.83 & 0.27 & -0.55 & 118.50 & 0.31 & $5.30 *$ & 111.20 & 1.32 & -2.08 & 16.90 & 0.90 & $18.86^{*}$ \\
\hline 49 & PAIG 346 x AKA 8 & 70.66 & 1.12 & -0.33 & 115.33 & 2.73 & -0.52 & 110.93 & 1.35 & -3.55 & 25.27 & 1.25 & -2.62 \\
\hline 50 & PAIG 346 x PhuleDhanwantary & 76.33 & 1.66 & -0.68 & 117.66 & 2.76 & -1.09 & 97.33 & 0.46 & -6.32 & 17.97 & 1.61 & -1.49 \\
\hline 51 & PAIG 346 x CNA 449 & 73.50 & 1.10 & -0.49 & 118.50 & 0.93 & -0.96 & 119.23 & 1.95 & 0.31 & 25.57 & 1.85 & -2.58 \\
\hline 52 & PAIG 346 x HD 514 & 71.50 & -1.39 & -0.65 & 115.33 & -0.90 & -0.77 & 99.80 & 1.03 & 9.42 & 25.67 & 0.96 & -1.67 \\
\hline 53 & PAIG 346 x DWDa 1402 & 70.83 & 1.94 & -0.42 & 115.66 & 0.69 & -1.16 & 121.33 & 1.43 & -5.88 & 27.90 & 0.23 & -1.84 \\
\hline 54 & PAIG 346 x JLA 794 & 73.00 & 1.10 & -0.49 & 117.66 & 1.83 & -1.14 & 123.23 & 0.85 & -6.29 & 27.03 & 0.21 & -2.55 \\
\hline 55 & PAIG 346 x Digvijay & 72.83 & 0.84 & 0.30 & 117.66 & 1.10 & 0.59 & 119.83 & 1.49 & -2.36 & 17.47 & 1.43 & -2.06 \\
\hline \multirow[t]{2}{*}{56} & PAIG 346 x G.Cot 23 & 73.83 & 1.37 & 0.02 & 118.66 & -0.65 & -0.15 & 116.56 & 0.84 & -9.16 & 18.93 & 0.87 & $17.66^{*}$ \\
\hline & Check & & & & & & & & & & & & \\
\hline 1 & PKVDH 1 & 72.33 & 0.84 & 0.30 & 117.66 & 0.90 & -0.77 & 104.83 & 1.01 & -5.83 & 16.18 & 0.15 & -2.58 \\
\hline 2 & PKV Suvarna & 72.66 & 2.22 & -0.68 & 118.00 & 0.21 & -0.68 & 105.83 & 0.41 & 14.26 & 17.53 & 0.20 & -2.61 \\
\hline 3 & NACH 12 & 74.66 & 0.54 & -0.11 & 119.16 & 0.69 & -1.16 & 109.13 & 0.76 & 4.57 & 18.53 & $0.29 *$ & -2.68 \\
\hline \multirow[t]{3}{*}{4} & PA 255 & 72.83 & 0.27 & -0.55 & 119.00 & -0.21 & -0.68 & 107.13 & 1.20 & 3.86 & 18.00 & 0.18 & -2.58 \\
\hline & Population Mean & 72.20 & & & 117.40 & & & 108.80 & & & 19.13 & & \\
\hline & S.E. $(\mathbf{m}) \pm$ & 0.52 & & & 0.70 & & & 1.4 & & & 1.46 & & \\
\hline
\end{tabular}


Table.1 (Contd...)

\begin{tabular}{|c|c|c|c|c|c|c|c|c|c|c|c|c|c|}
\hline \multirow{2}{*}{$\begin{array}{l}\text { Sr. } \\
\text { No. }\end{array}$} & \multirow[t]{2}{*}{ Genotype } & \multicolumn{3}{|c|}{ Number of bolls /plant } & \multicolumn{3}{|c|}{ Boll weight (g) } & \multicolumn{3}{|c|}{ Seed cotton yield/plant (g) } & \multicolumn{3}{|c|}{ Seed index (g) } \\
\hline & & Mean & Bi & $S^{2}$ di & Mean & Bi & $S^{2} \mathbf{d i}$ & Mean & Bi & $\mathbf{S}^{2} \mathrm{di}$ & Mean & Bi & $S^{2}$ di \\
\hline & Lines & & & & & & & & & & & & \\
\hline 1 & PA 801 & 23.50 & 0.95 & -3.75 & 2.62 & 2.63 & -0.0018 & 54.47 & $0.83 *$ & -14.70 & 5.91 & 0.57 & -0.0208 \\
\hline 2 & PA 740 & 24.00 & $-0.20 *$ & -3.83 & 2.69 & 1.89 & -0.0024 & 52.15 & 0.49 & -8.59 & 5.97 & -0.51 & -0.0124 \\
\hline 3 & PA 812 & 26.67 & 0.13 & -3.76 & 2.56 & 1.80 & -0.0026 & 60.88 & $0.18^{*}$ & -14.60 & 6.10 & 1.12 & -0.0077 \\
\hline 4 & PA 809 & 25.60 & 1.01 & -3.80 & 2.66 & 1.97 & -0.0012 & 58.30 & 0.67 & -14.66 & 6.40 & -1.75 & -0.0215 \\
\hline 5 & PA 785 & 24.23 & 0.78 & -3.83 & 2.61 & -2.46 & -0.0026 & 60.67 & 0.70 & -14.53 & 5.66 & 1.88 & -0.0210 \\
\hline 6 & PA 832 & 21.87 & 0.76 & -3.8 & 2.67 & -0.49 & -0.0006 & 52.25 & 0.71 & -14.67 & 6.00 & 2.15 & -0.0212 \\
\hline \multirow[t]{2}{*}{7} & PAIG 346 & 23.60 & $0.73 *$ & -3.84 & 2.73 & -0.17 & -0.0018 & 59.28 & $0.61 *$ & -14.69 & 6.47 & -0.53 & -0.0087 \\
\hline & Testers & & & & & & & & & & & & \\
\hline 1 & AKA 8 & 24.33 & 0.63 & -3.51 & 2.37 & 0.17 & -0.0006 & 56.57 & $0.54 *$ & -14.67 & 5.70 & 1.20 & -0.0198 \\
\hline 2 & PhuleDhanwantary & 19.33 & 0.30 & -3.80 & 2.27 & -0.81 & $0.0114 *$ & 39.30 & 0.42 & -14.46 & 5.66 & -0.13 & -0.0113 \\
\hline 3 & CNA 449 & 21.93 & 0.48 & -3.65 & 2.61 & -1.96 & 0.0002 & 47.20 & 0.35 & -14.24 & 6.46 & -0.10 & -0.0143 \\
\hline 4 & HD 514 & 25.90 & 0.26 & -3.77 & 2.49 & 0.00 & -0.0012 & 55.75 & 0.49 & -14.06 & 5.44 & 0.47 & -0.0159 \\
\hline 5 & DWDa 1402 & 22.47 & 0.51 & -3.78 & 2.67 & 2.46 & -0.0006 & 49.28 & $0.55 *$ & -14.65 & 5.93 & 2.66 & -0.0226 \\
\hline 6 & JLA 794 & 19.47 & 0.30 & -3.79 & 2.37 & 0.17 & -0.0026 & 47.43 & 0.44 & -14.39 & 5.70 & 1.20 & -0.0210 \\
\hline 7 & Digvijay & 19.30 & 0.20 & -2.84 & 2.27 & -0.81 & 0.0042 & 34.38 & 0.34 & -14.54 & 5.66 & -0.13 & -0.0183 \\
\hline \multirow[t]{2}{*}{8} & G.Cot 23 & 18.10 & 0.59 & -0.28 & 2.61 & -1.96 & 0.0054 & 34.52 & 0.44 & 3.73 & 6.46 & -0.10 & -0.0204 \\
\hline & Crosses & & & & & & & & & & & & \\
\hline 1 & PA 801 x AKA 8 & 29.80 & 1.00 & -1.34 & 2.72 & 2.62 & -0.0028 & 71.03 & 1.49 & -8.65 & 6.06 & 0.19 & -0.0157 \\
\hline 2 & PA 801 x PhuleDhanwantary & 22.93 & 1.30 & -1.81 & 2.74 & -0.33 & -0.0022 & 55.40 & 1.64 & -9.41 & 5.88 & 0.30 & -0.0146 \\
\hline 3 & PA 801 x CNA 449 & 28.50 & 1.08 & -2.27 & 2.87 & 4.75 & 0.0030 & 71.47 & 1.40 & -12.28 & 6.85 & -0.31 & -0.0117 \\
\hline 4 & PA 801 x HD 514 & 33.47 & 0.95 & -3.58 & 2.75 & 1.97 & -0.0022 & 74.67 & 1.06 & -13.68 & 5.96 & 2.15 & -0.0209 \\
\hline 5 & PA 801 x DWDa 1402 & 28.97 & 1.57 & -3.45 & 2.82 & 0.33 & -0.0012 & 66.93 & 1.11 & -13.11 & 6.26 & -0.39 & -0.0132 \\
\hline 6 & PA 801 x JLA 794 & 30.20 & 0.68 & $17.03^{*}$ & 2.80 & 1.47 & 0.0010 & 69.43 & 0.69 & 38.05 & 6.91 & -0.27 & -0.0211 \\
\hline 7 & PA 801 x Digvijay & 20.87 & 1.85 & 0.83 & 2.64 & -1.31 & -0.0028 & 47.40 & 2.00 & -13.67 & 6.43 & $9.27 *$ & -0.0232 \\
\hline 8 & PA 801 x G.Cot 23 & 21.30 & 0.99 & $27.89 * *$ & 2.68 & 1.48 & -0.0026 & 49.13 & 0.83 & $59.68 *$ & 6.65 & 1.97 & -0.0212 \\
\hline 9 & PA 740 x AKA 8 & 27.03 & 1.49 & -2.77 & 2.66 & 2.30 & -0.0028 & 60.33 & 1.65 & -10.26 & 6.00 & -3.12 & -0.0111 \\
\hline 10 & PA 740 x PhuleDhanwantary & 19.53 & 1.33 & -2.11 & 2.68 & -1.47 & -0.0018 & 46.13 & 1.54 & -13.10 & 5.93 & 2.25 & -0.0186 \\
\hline
\end{tabular}


Table.1 (Contd...)

\begin{tabular}{|c|c|c|c|c|c|c|c|c|c|c|c|c|c|}
\hline \multirow{2}{*}{$\begin{array}{l}\text { Sr. } \\
\text { No. }\end{array}$} & \multirow[t]{2}{*}{ Genotype } & \multicolumn{3}{|c|}{ Number of bolls /plant } & \multicolumn{3}{|c|}{ Boll weight (g) } & \multicolumn{3}{|c|}{ Seed cotton yield/plant (g) } & \multicolumn{3}{|c|}{ Seed index (g) } \\
\hline & & Mean & $\mathbf{B i}$ & $\mathrm{S}^{2} \mathrm{di}$ & Mean & Bi & $S^{2}$ di & Mean & Bi & $S^{2} d i$ & Mean & $\mathbf{B i}$ & $S^{2}$ di \\
\hline 11 & PA 740 x CNA 449 & 25.63 & 1.46 & -3.25 & 2.82 & 2.63 & -0.0022 & 64.90 & 1.22 & -13.28 & 6.98 & 5.11 & 0.0013 \\
\hline 12 & PA 740 x HD 514 & 30.43 & 0.54 & 10.87 & 2.72 & 1.80 & -0.0026 & 68.58 & 0.46 & $56.09 *$ & 6.13 & 1.17 & -0.0226 \\
\hline 13 & PA 740 x DWDa 1402 & 27.27 & 1.29 & -2.37 & 2.86 & -0.66 & 0.0010 & 64.80 & 1.15 & -13.41 & 6.68 & -0.10 & -0.0143 \\
\hline 14 & PA 740 x JLA 794 & 21.90 & 0.84 & 11.04 & 2.89 & 2.29 & -0.0012 & 53.70 & 0.77 & $63.50 *$ & 7.11 & 0.21 & -0.0166 \\
\hline 15 & PA 740 x Digvijay & 27.53 & 1.57 & -2.67 & 2.98 & 0.49 & -0.0018 & 65.73 & 1.21 & -10.82 & 7.03 & 0.07 & 0.0025 \\
\hline 16 & PA 740 x G.Cot 23 & 26.47 & 1.62 & -1.88 & 2.88 & -0.16 & 0.0010 & 60.83 & 1.51 & -5.12 & 6.81 & 0.17 & -0.0178 \\
\hline 17 & PA 812 x AKA 8 & 28.63 & 1.49 & -2.77 & 2.71 & -1.97 & -0.0022 & 70.43 & 1.29 & -13.34 & 6.36 & 2.14 & -0.0217 \\
\hline 18 & PA 812 x PhuleDhanwantary & 23.73 & 1.49 & -2.77 & 2.63 & -4.76 & -0.0026 & 51.60 & 1.46 & -8.98 & 6.06 & -1.29 & 0.0455 \\
\hline 19 & PA 812 x CNA 449 & 29.03 & 0.90 & $18.93 *$ & 2.77 & 2.46 & -0.0006 & 68.87 & 0.67 & 22.87 & 6.88 & 2.57 & 0.0153 \\
\hline 20 & PA 812 x HD 514 & 30.30 & -0.25 & 10.22 & 2.68 & 1.97 & -0.0022 & 69.70 & 0.35 & 11.63 & 6.67 & 3.66 & -0.0229 \\
\hline 21 & PA 812 x DWDa 1402 & 25.30 & 1.43 & -2.06 & 2.80 & -2.95 & -0.0012 & 63.18 & 1.18 & -14.43 & 6.51 & 2.40 & -0.0222 \\
\hline 22 & PA 812 x JLA 794 & 25.80 & 1.08 & $12.88^{*}$ & 2.80 & 2.13 & 0.0030 & 63.83 & 1.02 & $81.68 *$ & 6.95 & 7.24 & -0.0188 \\
\hline 23 & PA 812 x Digvijay & 24.20 & 1.62 & -2.10 & 2.76 & $14.26^{*}$ & -0.0030 & 55.07 & 1.57 & -2.05 & 6.77 & -7.31 & -0.0187 \\
\hline 24 & PA 812 x G.Cot 23 & 25.13 & 0.99 & $24.67 * *$ & 2.67 & -2.46 & -0.0018 & 60.57 & 0.87 & $73.93 *$ & 6.60 & 2.17 & -0.0215 \\
\hline 25 & PA 809 x AKA 8 & 36.60 & 0.22 & 3.65 & 2.71 & 4.18 & -0.0020 & 84.50 & 0.44 & -14.44 & 6.50 & 1.32 & -0.0212 \\
\hline 26 & PA 809 x PhuleDhanwantary & 24.00 & 1.66 & -2.90 & 2.66 & -1.80 & -0.0026 & 55.72 & 1.62 & -3.40 & 6.24 & -0.47 & -0.0146 \\
\hline 27 & PA 809 x CNA 449 & 33.43 & 1.22 & -3.78 & 2.81 & 1.97 & -0.0022 & 73.97 & $0.48 *$ & -14.64 & 7.19 & 1.99 & -0.0205 \\
\hline 28 & PA 809 x HD 514 & 30.23 & 1.25 & -3.04 & 2.74 & 1.97 & -0.0028 & 65.23 & 1.12 & -14.42 & 6.46 & $11.65^{*}$ & -0.0224 \\
\hline 29 & PA 809 x DWDa 1402 & 27.87 & 1.35 & -1.97 & 2.85 & 2.95 & -0.0022 & 68.53 & 1.57 & -12.70 & 6.77 & -2.04 & $0.160 * *$ \\
\hline 30 & PA 809 x JLA 794 & 29.30 & 1.50 & -2.28 & 2.91 & 2.95 & -0.0022 & 70.50 & 1.42 & -2.63 & 7.17 & -1.90 & -0.0115 \\
\hline 31 & PA 809 x Digvijay & 21.93 & 1.24 & $15.95 *$ & 2.687 & 1.80 & -0.0030 & 53.67 & 2.32 & -9.24 & 6.37 & 2.28 & -0.0199 \\
\hline 32 & PA 809 x G.Cot 23 & 28.27 & 1.64 & -3.55 & 2.82 & -0.01 & 0.0042 & 60.88 & 1.59 & -2.20 & 5.95 & 2.60 & -0.0200 \\
\hline 33 & PA $785 \times$ AKA 8 & 29.50 & 1.31 & -3.05 & 2.64 & 0.49 & 0.0010 & 68.53 & 1.13 & -7.72 & 6.35 & 1.07 & -0.0181 \\
\hline 34 & PA $785 \times$ PhuleDhanwantary & 28.50 & 1.42 & -2.40 & 2.66 & 4.58 & 0.0020 & 62.60 & 1.14 & -12.48 & 6.09 & 2.37 & -0.0200 \\
\hline 35 & PA 785 x CNA 449 & 34.57 & 1.17 & -3.69 & 2.80 & 2.30 & -0.0012 & 79.43 & 1.16 & -14.07 & 6.56 & 0.17 & -0.0128 \\
\hline 36 & PA 785 x HD 514 & 23.87 & 1.64 & -1.06 & 2.72 & -2.46 & -0.0018 & 57.22 & 1.45 & -7.98 & 5.96 & -2.94 & 0.0531 \\
\hline 37 & PA 785 x DWDa 1402 & 26.43 & 1.64 & -0.79 & 2.717 & 2.29 & -0.0022 & 62.62 & 1.68 & -4.32 & 5.87 & 0.26 & -0.0101 \\
\hline 38 & PA 785 x JLA 794 & 28.77 & 1.01 & $20.30 *$ & 2.81 & 5.24 & 0.0002 & 65.87 & 1.17 & $138.11^{* *}$ & 6.60 & -0.20 & -0.0131 \\
\hline 39 & PA 785 x Digvijay & 23.43 & 0.88 & $11.48 *$ & 2.56 & 0.49 & -0.0018 & 56.10 & 1.04 & $84.50 *$ & 6.50 & -5.11 & -0.0165 \\
\hline 40 & PA $785 \times$ G.Cot 23 & 23.75 & 1.48 & 0.11 & 2.77 & -2.63 & -0.0012 & 54.97 & 1.92 & 4.35 & 6.31 & -6.58 & 0.0586 \\
\hline
\end{tabular}


Table.1 (Contd...)

\begin{tabular}{|c|c|c|c|c|c|c|c|c|c|c|c|c|c|}
\hline \multirow{2}{*}{$\begin{array}{l}\text { Sr. } \\
\text { No. }\end{array}$} & \multirow[t]{2}{*}{ Genotype } & \multicolumn{3}{|c|}{ Number of bolls /plant } & \multicolumn{3}{|c|}{ Boll weight (g) } & \multicolumn{3}{|c|}{ Seed cotton yield/plant (g) } & \multicolumn{3}{|c|}{ Seed index (g) } \\
\hline & & Mean & $\mathbf{B i}$ & $S^{2} \mathrm{di}$ & Mean & $\mathbf{B i}$ & $S^{2} \mathrm{di}$ & Mean & $\mathbf{B i}$ & $S^{2} \mathrm{di}$ & Mean & $\mathbf{B i}$ & $S^{2} \mathrm{di}$ \\
\hline 41 & PA $832 \times$ AKA 8 & 24.63 & 0.69 & $11.37 *$ & 2.66 & 2.79 & -0.0018 & 52.77 & 0.73 & 26.86 & 6.37 & -0.99 & -0.0218 \\
\hline 42 & PA 832 x PhuleDhanwantary & 19.90 & 0.71 & $16.57^{*}$ & 2.54 & -0.75 & 0.0075 & 44.97 & 0.42 & 26.01 & 5.90 & 3.77 & 0.0106 \\
\hline 43 & PA 832 x CNA 449 & 29.08 & 0.98 & -3.85 & 2.81 & -0.50 & 0.0082 & 70.57 & 0.55 & -12.34 & 6.76 & -0.17 & -0.0156 \\
\hline 44 & PA $832 \times$ HD 514 & 21.47 & 1.30 & -1.32 & 2.61 & -0.01 & 0.0020 & 51.73 & 1.22 & -8.57 & 6.15 & 2.76 & -0.0212 \\
\hline 45 & PA 832 x DWDa 1402 & 25.70 & 1.30 & -1.57 & 2.83 & 0.82 & -0.0006 & 57.00 & 1.30 & -13.09 & 6.52 & 2.30 & -0.0210 \\
\hline 46 & PA 832 x JLA 794 & 27.50 & 1.24 & -2.02 & 2.81 & 1.15 & -0.0018 & 62.40 & 1.32 & -10.09 & 7.28 & 2.97 & -0.0220 \\
\hline 47 & PA 832 x Digvijay & 17.47 & 1.13 & -1.62 & 2.60 & -0.16 & -0.0018 & 43.57 & 1.38 & -3.98 & 6.26 & 0.44 & -0.0103 \\
\hline 48 & PA 832 x G.Cot 23 & 22.37 & 1.04 & $21.38^{*}$ & 2.78 & -2.30 & -0.0022 & 49.47 & 1.06 & $59.73^{*}$ & 6.73 & 2.69 & -0.0174 \\
\hline 49 & PAIG 346 x AKA 8 & 32.00 & 0.86 & -3.78 & 2.71 & 1.80 & -0.0026 & 74.90 & 0.85 & -14.45 & 6.56 & -0.19 & 0.0048 \\
\hline 50 & PAIG 346 x PhuleDhanwantary & 23.23 & 1.61 & -3.71 & 2.64 & 2.79 & -0.0018 & 51.50 & 0.83 & -14.33 & 5.93 & 2.94 & -0.0195 \\
\hline 51 & PAIG 346 x CNA 449 & 31.90 & 1.41 & -3.64 & 2.95 & 1.31 & -0.0028 & 78.50 & 0.98 & -14.56 & 7.20 & 0.00 & 0.0046 \\
\hline 52 & PAIG 346 x HD 514 & 31.53 & 1.39 & -3.78 & 2.78 & 0.32 & 0.0042 & 77.17 & 1.41 & -12.62 & 6.36 & 0.04 & -0.0178 \\
\hline 53 & PAIG 346 x DWDa 1402 & 35.73 & 1.07 & 0.96 & 2.81 & -1.80 & -0.0030 & 84.80 & 1.20 & -9.49 & 7.01 & 2.49 & -0.0193 \\
\hline 54 & PAIG 346 x JLA 794 & 34.63 & 0.76 & -2.87 & 2.90 & 3.11 & -0.0030 & 85.00 & 1.01 & -14.12 & 7.40 & -0.90 & -0.0217 \\
\hline 55 & PAIG 346 x Digvijay & 19.57 & 1.35 & -1.97 & 2.85 & 8.03 & -0.0026 & 45.13 & 1.79 & -5.89 & 6.32 & $7.79 *$ & -0.0228 \\
\hline \multirow[t]{2}{*}{56} & PAIG 346 x G.Cot 23 & 23.00 & 0.67 & $34.99 * *$ & 2.86 & 0.16 & 0.0054 & 53.80 & 0.41 & $163.28 * *$ & 7.03 & 2.06 & -0.0229 \\
\hline & Check & & & & & & & & & & & & \\
\hline 1 & PKVDH 1 & 23.83 & 0.28 & -3.41 & 2.37 & 1.31 & -0.0028 & 57.53 & $0.34^{*}$ & -14.68 & 5.73 & 0.93 & -0.0207 \\
\hline 2 & PKV Suvarna & 24.63 & 0.14 & -3.60 & 2.54 & -1.97 & -0.0022 & 63.80 & 0.14 & -14.32 & 6.05 & -0.30 & -0.0175 \\
\hline 3 & NACH 12 & 26.00 & 0.11 & -2.72 & 2.69 & 2.79 & -0.0018 & 66.50 & 0.41 & -14.57 & 6.09 & 3.29 & -0.0229 \\
\hline \multirow[t]{3}{*}{4} & PA 255 & 22.90 & 0.60 & -3.26 & 2.63 & 1.80 & -0.0026 & 60.52 & 0.51 & -14.43 & 6.19 & -1.92 & -0.0220 \\
\hline & Population Mean & 25.89 & & & 2.72 & & & 60.37 & & & 6.41 & & \\
\hline & S.E. $(\mathrm{m}) \pm$ & 1.63 & & & 0.03 & & & 2.97 & & & 0.07 & & \\
\hline
\end{tabular}


Table.1 (Contd...)

\begin{tabular}{|c|c|c|c|c|c|c|c|c|c|c|c|c|c|}
\hline \multirow{2}{*}{$\begin{array}{l}\text { Sr. } \\
\text { No. }\end{array}$} & \multirow[t]{2}{*}{ Genotype } & \multicolumn{3}{|c|}{ Lint index (g) } & \multicolumn{3}{|c|}{ Harvest index (\%) } & \multicolumn{3}{|c|}{ Ginning percentage (\%) } & \multicolumn{3}{|c|}{ Days to maturity } \\
\hline & & Mean & Bi & $S^{2}$ di & Mean & Bi & $\mathbf{S}^{2} \mathrm{di}$ & Mean & $\mathbf{B i}$ & $\mathbf{S}^{2} \mathrm{di}$ & Mean & Bi & $S^{2}$ di \\
\hline & Lines & & & & & & & & & & & & \\
\hline 1 & PA 801 & 3.53 & 1.85 & -0.004 & 35.37 & 0.81 & -1.34 & 36.65 & 2.21 & -0.64 & 155.83 & 1.52 & -0.82 \\
\hline 2 & PA 740 & 3.60 & 1.46 & -0.006 & 34.86 & 0.37 & -1.18 & 36.73 & 1.45 & -0.40 & 158.16 & 0.81 & -1.40 \\
\hline 3 & PA 812 & 3.43 & 2.56 & -0.007 & 36.78 & 0.73 & -1.28 & 34.74 & -2.68 & -0.50 & 156.33 & 0.35 & -1.27 \\
\hline 4 & PA 809 & 3.54 & 2.56 & -0.007 & 36.28 & 0.72 & -1.31 & 36.44 & 0.78 & -0.66 & 157.33 & 1.73 & -0.99 \\
\hline 5 & PA 785 & 3.53 & 0.26 & -0.007 & 35.69 & 0.64 & -1.35 & 35.94 & 3.20 & -0.65 & 158.33 & 1.62 & -1.40 \\
\hline 6 & PA 832 & 3.44 & 1.46 & -0.006 & 34.47 & $0.46^{*}$ & -1.35 & 34.79 & 1.80 & -0.62 & 157.16 & 0.81 & -1.40 \\
\hline \multirow[t]{2}{*}{7} & PAIG 346 & 3.68 & 1.59 & -0.006 & 35.65 & 0.19 & -1.28 & 35.98 & 2.61 & -0.54 & 158.00 & 1.16 & -1.25 \\
\hline & Testers & & & & & & & & & & & & \\
\hline 1 & AKA 8 & 3.69 & 0.46 & -0.005 & 35.21 & 0.64 & -1.29 & 37.34 & 0.82 & -0.13 & 155.50 & 1.16 & -1.25 \\
\hline 2 & PhuleDhanwantary & 3.43 & 1.53 & -0.006 & 33.70 & $0.53 *$ & -1.35 & 35.71 & 0.96 & -0.62 & 156.50 & 1.27 & -1.31 \\
\hline 3 & CNA 449 & 3.59 & 0.79 & -0.005 & 35.31 & 0.58 & -1.32 & 35.88 & -0.92 & -0.65 & 158.16 & 3.24 & -1.39 \\
\hline 4 & HD 514 & 3.73 & 0.79 & -0.005 & 35.52 & 0.64 & -1.28 & 37.54 & -1.15 & 0.34 & 156.00 & 1.16 & -1.25 \\
\hline 5 & DWDa 1402 & 3.58 & 1.19 & -0.007 & 36.24 & 0.41 & -1.33 & 35.33 & -1.96 & -0.46 & 156.16 & 2.08 & -1.33 \\
\hline 6 & JLA 794 & 3.56 & 1.79 & -0.007 & 35.95 & 0.51 & -1.16 & 36.26 & -1.48 & -0.65 & 157.33 & -0.70 & -0.86 \\
\hline 7 & Digvijay & 3.69 & -2.44 & -0.007 & 33.18 & 0.43 & -1.33 & 36.66 & 3.21 & -0.66 & 169.00 & 2.54 & -1.03 \\
\hline \multirow[t]{2}{*}{8} & G.Cot 23 & 3.88 & $0.00 *$ & -0.007 & 31.76 & 0.20 & -1.27 & 36.75 & 0.79 & -0.60 & 167.33 & 1.62 & -1.40 \\
\hline & Crosses & & & & & & & & & & & & \\
\hline 1 & PA 801 x AKA 8 & 3.90 & $-3.44 *$ & -0.007 & 37.57 & 0.07 & -0.86 & 38.63 & 0.46 & -0.55 & 150.66 & 2.08 & -1.33 \\
\hline 2 & PA 801 x PhuleDhanwantary & 3.65 & 1.68 & -0.004 & 36.01 & 0.84 & -0.75 & 37.56 & 5.00 & -0.50 & 154.50 & 2.43 & -1.40 \\
\hline 3 & PA 801 x CNA 449 & 3.68 & 1.74 & -0.007 & 39.30 & 1.74 & -0.71 & 37.37 & -3.79 & 0.12 & 158.00 & 2.22 & 0.85 \\
\hline 4 & PA 801 x HD 514 & 3.89 & 1.12 & -0.003 & 41.37 & 2.34 & -0.01 & 38.35 & -1.50 & -0.54 & 154.00 & 1.16 & -1.25 \\
\hline 5 & PA 801 x DWDa 1402 & 3.64 & -0.52 & -0.005 & 37.82 & 0.95 & -1.25 & 36.93 & 2.45 & -0.63 & 153.66 & 2.29 & 1.44 \\
\hline 6 & PA 801 x JLA 794 & 3.65 & 2.57 & -0.006 & 38.87 & 0.95 & -1.32 & 35.57 & -2.90 & -0.66 & 158.33 & 1.52 & -0.82 \\
\hline 7 & PA 801 x Digvijay & 3.69 & 2.96 & -0.007 & 35.74 & 1.30 & -0.69 & 36.68 & -1.94 & -0.49 & 163.83 & 1.62 & -1.40 \\
\hline 8 & PA 801 x G.Cot 23 & 3.91 & -0.22 & -0.007 & 35.96 & 1.47 & -1.10 & 37.69 & -2.74 & -0.45 & 164.33 & 2.78 & -1.19 \\
\hline 9 & PA 740 x AKA 8 & 3.65 & 0.47 & -0.006 & 37.44 & 1.67 & -0.66 & 39.98 & 3.53 & -0.35 & 155.33 & 0.46 & -1.29 \\
\hline 10 & PA 740 x PhuleDhanwantary & 3.69 & -0.45 & -0.005 & 36.44 & 1.09 & -1.11 & 38.01 & -2.57 & -0.67 & 157.00 & 1.16 & -1.25 \\
\hline
\end{tabular}


Table.1 (Contd...)

\begin{tabular}{|c|c|c|c|c|c|c|c|c|c|c|c|c|c|}
\hline \multirow{2}{*}{$\begin{array}{l}\text { Sr. } \\
\text { No. }\end{array}$} & \multirow[t]{2}{*}{ Genotype } & \multicolumn{3}{|c|}{ Lint index (g) } & \multicolumn{3}{|c|}{ Harvest index (\%) } & \multicolumn{3}{|c|}{ Ginning percentage (\%) } & \multicolumn{3}{|c|}{ Days to maturity } \\
\hline & & Mean & $\mathbf{B i}$ & $S^{2} \mathbf{d i}$ & Mean & Bi & $S^{2} d i$ & Mean & Bi & $S^{2} d i$ & Mean & $\mathbf{B i}$ & $S^{2} d i$ \\
\hline 11 & PA 740 x CNA 449 & 3.69 & -0.15 & -0.007 & 38.38 & 0.59 & -1.11 & 37.60 & -1.52 & -0.57 & 157.33 & 1.52 & -0.82 \\
\hline 12 & PA 740 x HD 514 & 3.85 & -0.84 & -0.006 & 40.07 & 1.21 & -0.19 & 40.23 & -1.00 & -0.67 & 153.66 & 2.19 & -0.44 \\
\hline 13 & PA 740 x DWDa 1402 & 3.59 & 0.97 & -0.007 & 38.59 & 1.15 & -1.32 & 36.81 & -2.59 & -0.51 & 155.50 & 1.37 & -0.38 \\
\hline 14 & PA 740 x JLA 794 & 3.72 & 0.36 & -0.005 & 37.37 & $1.42 *$ & -1.35 & 36.50 & -2.51 & -0.64 & 156.16 & 2.08 & -1.33 \\
\hline 15 & PA 740 x Digvijay & 3.83 & -0.25 & -0.007 & 40.46 & 0.82 & -1.34 & 38.13 & 1.25 & -0.19 & 159.33 & 2.89 & -1.34 \\
\hline 16 & PA 740 x G.Cot 23 & 3.86 & 1.23 & -0.007 & 39.56 & 2.18 & -1.12 & 37.46 & 0.88 & -0.31 & 158.33 & 0.25 & -0.25 \\
\hline 17 & PA 812 x AKA 8 & 3.60 & 0.72 & -0.006 & 39.33 & 1.81 & -1.33 & 38.59 & 2.69 & -0.10 & 153.33 & 0.25 & -0.25 \\
\hline 18 & PA 812 x PhuleDhanwantary & 3.73 & 3.83 & -0.006 & 37.37 & 0.79 & -1.33 & 37.75 & 4.34 & -0.19 & 154.33 & 0.56 & -0.32 \\
\hline 19 & PA 812 x CNA 449 & 3.63 & 0.79 & -0.005 & 38.60 & 0.60 & -1.35 & 36.37 & 0.61 & -0.66 & 155.50 & 1.37 & -0.38 \\
\hline 20 & PA 812 x HD 514 & 4.11 & -3.92 & -0.007 & 39.28 & $0.48 *$ & -1.35 & 37.69 & 1.62 & -0.43 & 152.00 & 2.43 & -1.40 \\
\hline 21 & PA 812 x DWDa 1402 & 3.65 & -2.04 & -0.007 & 37.95 & 0.96 & -1.20 & 35.60 & 0.70 & -0.61 & 155.33 & 0.46 & -1.29 \\
\hline 22 & PA 812 x JLA 794 & 3.84 & 0.49 & -0.007 & 37.82 & -0.22 & -1.17 & 37.63 & -0.09 & -0.57 & 156.33 & 1.83 & 0.42 \\
\hline 23 & PA 812 x Digvijay & 3.64 & 1.46 & -0.006 & 36.47 & 0.58 & -0.71 & 37.14 & 2.73 & -0.67 & 155.50 & 2.54 & -1.03 \\
\hline 24 & PA 812 x G.Cot 23 & 3.83 & 1.36 & -0.004 & 35.37 & 1.10 & -1.10 & 37.24 & 2.38 & -0.58 & 158.66 & -0.56 & -0.32 \\
\hline 25 & PA 809 x AKA 8 & 3.81 & 2.92 & -0.005 & 43.73 & 1.32 & -1.31 & 38.79 & 4.16 & -0.55 & 155.83 & 0.46 & -1.29 \\
\hline 26 & PA 809 x PhuleDhanwantary & 3.71 & -0.63 & -0.007 & 36.33 & 1.13 & -1.30 & 37.79 & 3.11 & 0.01 & 155.16 & 2.40 & $4.31 *$ \\
\hline 27 & PA 809 x CNA 449 & 3.58 & -2.11 & -0.007 & 42.13 & 0.91 & 1.09 & 37.31 & -0.07 & 0.51 & 159.66 & 0.60 & 0.67 \\
\hline 28 & PA 809 x HD 514 & 3.89 & 2.86 & -0.007 & 37.38 & 0.93 & -1.33 & 39.27 & 1.30 & -0.47 & 154.00 & -3.59 & -1.16 \\
\hline 29 & PA 809 x DWDa 1402 & 3.61 & 0.97 & -0.007 & 38.49 & 1.51 & -0.96 & 37.83 & -5.09 & -0.58 & 154.33 & $-4.51 *$ & -1.37 \\
\hline 30 & PA 809 x JLA 794 & 3.64 & -0.54 & -0.006 & 39.78 & 0.87 & 0.10 & 39.44 & -1.12 & -0.41 & 156.66 & 1.97 & -1.22 \\
\hline 31 & PA 809 x Digvijay & 3.70 & 1.20 & -0.005 & 36.52 & 1.58 & 0.84 & 36.92 & -2.64 & -0.46 & 159.00 & 4.76 & -0.61 \\
\hline 32 & PA 809 x G.Cot 23 & 3.73 & 1.53 & -0.004 & 38.07 & 2.10 & -1.25 & 38.33 & 1.88 & -0.63 & 158.66 & 3.35 & -1.07 \\
\hline 33 & PA 785 x AKA 8 & 3.84 & -2.94 & -0.007 & 38.90 & 1.23 & -1.34 & 39.87 & 1.36 & -0.41 & 153.16 & 0.70 & -0.86 \\
\hline 34 & PA 785 x PhuleDhanwantary & 3.68 & 1.17 & -0.007 & 37.05 & 0.64 & -1.33 & 37.36 & 2.11 & -0.65 & 156.50 & 1.16 & -1.25 \\
\hline 35 & PA 785 x CNA 449 & 3.89 & $15.80 * *$ & 0.075 & 42.64 & 1.06 & 1.68 & 38.83 & 9.59 & -0.29 & 157.33 & 2.68 & -0.05 \\
\hline 36 & PA 785 x HD 514 & 3.85 & -0.47 & -0.006 & 37.16 & 1.57 & -1.26 & 39.03 & 0.68 & 0.80 & 154.16 & 0.92 & -0.95 \\
\hline 37 & PA 785 x DWDa 1402 & 3.58 & 2.81 & -0.006 & 38.16 & 0.72 & -1.34 & 34.27 & 2.61 & -0.66 & 157.00 & 1.27 & -1.31 \\
\hline 38 & PA 785 x JLA 794 & 3.73 & 1.70 & -0.006 & 38.21 & 1.74 & -1.07 & 36.58 & -1.42 & -0.54 & 159.83 & $-0.81 *$ & -1.40 \\
\hline 39 & PA 785 x Digvijay & 3.69 & -1.93 & -0.005 & 35.92 & 1.22 & -0.80 & 36.31 & 6.79 & -0.64 & 156.83 & -0.70 & -0.86 \\
\hline 40 & PA 785 x G.Cot 23 & 3.80 & 0.36 & -0.005 & 36.61 & 0.93 & -0.80 & 38.28 & 0.23 & -0.48 & 156.50 & 3.49 & 0.03 \\
\hline
\end{tabular}


Table.1 (Contd...)

\begin{tabular}{|c|c|c|c|c|c|c|c|c|c|c|c|c|c|}
\hline \multirow{2}{*}{$\begin{array}{l}\text { Sr. } \\
\text { No. }\end{array}$} & \multirow[t]{2}{*}{ Genotype } & \multicolumn{3}{|c|}{ Lint index (g) } & \multicolumn{3}{|c|}{ Harvest index (\%) } & \multicolumn{3}{|c|}{ Ginning percentage ( $\%$ ) } & \multicolumn{3}{|c|}{ Days to maturity } \\
\hline & & Mean & $\mathbf{B i}$ & $\mathbf{S}^{2} \mathrm{di}$ & Mean & $\mathbf{B i}$ & $S^{2} d i$ & Mean & $\mathbf{B i}$ & $S^{2}$ di & Mean & $\mathbf{B i}$ & $S^{2}$ di \\
\hline 41 & PA $832 \times$ AKA 8 & 3.70 & 2.98 & -0.002 & 36.58 & 0.99 & -0.89 & 37.41 & 2.89 & -0.56 & 152.83 & 0.35 & -1.27 \\
\hline 42 & PA 832 x PhuleDhanwantary & 3.52 & 2.60 & -0.006 & 35.57 & 0.83 & -1.34 & 36.77 & 1.40 & -0.53 & 155.00 & $0.00 *$ & -1.40 \\
\hline 43 & PA 832 x CNA 449 & 3.65 & 1.60 & -0.004 & 41.60 & 3.09 & -0.32 & 35.46 & 3.47 & -0.65 & 156.00 & 2.43 & -1.40 \\
\hline 44 & PA 832 x HD 514 & 3.72 & 2.77 & -0.006 & 37.14 & 0.99 & -1.17 & 38.97 & 3.12 & -0.57 & 151.83 & 5.22 & -1.10 \\
\hline 45 & PA 832 x DWDa 1402 & 3.61 & 2.96 & -0.005 & 37.32 & 1.15 & -1.34 & 36.16 & 4.41 & -0.64 & 155.00 & 0.21 & 0.58 \\
\hline 46 & PA 832 x JLA 794 & 3.61 & 1.03 & -0.004 & 37.68 & 0.97 & -0.72 & 35.92 & 3.77 & -0.19 & 156.00 & 2.43 & -1.40 \\
\hline 47 & PA 832 x Digvijay & 3.65 & -0.86 & -0.005 & 35.49 & 0.84 & -1.35 & 37.42 & 2.57 & -0.59 & 157.00 & -3.59 & -1.16 \\
\hline 48 & PA 832 x G.Cot 23 & 3.69 & 1.05 & -0.006 & 34.62 & -0.21 & -1.29 & 38.35 & 3.11 & 0.87 & 159.83 & -3.35 & -1.07 \\
\hline 49 & PAIG $346 \times$ AKA 8 & 3.78 & 0.95 & -0.004 & 41.58 & 1.82 & -0.67 & 37.35 & 0.82 & -0.65 & 155.33 & -0.70 & -0.86 \\
\hline 50 & PAIG 346 x PhuleDhanwantary & 3.71 & 3.16 & -0.005 & 36.81 & 0.81 & -1.34 & 38.52 & 1.92 & -0.62 & 156.16 & 0.81 & -1.40 \\
\hline 51 & PAIG 346 x CNA 449 & 3.70 & 0.26 & -0.007 & 42.08 & 1.68 & -1.12 & 37.02 & -0.04 & -0.58 & 159.83 & 0.25 & -0.25 \\
\hline 52 & PAIG 346 x HD 514 & 4.03 & 1.74 & -0.007 & 42.18 & 1.64 & 0.46 & 41.75 & -2.75 & -0.28 & 155.66 & -0.46 & -1.29 \\
\hline 53 & PAIG 346 x DWDa 1402 & 3.82 & 1.94 & -0.005 & 43.35 & 1.68 & -1.23 & 38.58 & 4.60 & -0.63 & 155.00 & 1.27 & -1.31 \\
\hline 54 & PAIG 346 x JLA 794 & 3.77 & 0.47 & -0.006 & 43.41 & 1.51 & -1.18 & 39.12 & 2.64 & 0.17 & 156.16 & -0.35 & -1.27 \\
\hline 55 & PAIG 346 x Digvijay & 3.79 & 0.41 & -0.007 & 37.29 & 1.77 & -1.28 & 35.31 & 1.16 & -0.41 & 158.33 & $-0.81 *$ & -1.40 \\
\hline \multirow[t]{2}{*}{56} & PAIG 346 x G.Cot 23 & 3.77 & -1.81 & -0.007 & 36.98 & 1.07 & -1.02 & 36.94 & 2.83 & -0.23 & 158.83 & $-4.51 *$ & -1.37 \\
\hline & Check & & & & & & & & & & & & \\
\hline 1 & PKVDH 1 & 3.60 & 3.60 & -0.007 & 35.70 & -0.06 & -1.17 & 37.64 & -1.68 & -0.55 & 157.66 & -0.35 & -1.27 \\
\hline 2 & PKV Suvarna & 3.50 & 0.68 & -0.006 & 36.67 & 0.58 & -1.34 & 35.87 & 1.46 & -0.61 & 158.83 & 1.73 & -0.99 \\
\hline 3 & NACH 12 & 3.74 & 2.42 & -0.004 & 37.14 & $0.27 *$ & -1.35 & 37.66 & 0.17 & -0.64 & 158.83 & 1.52 & -0.82 \\
\hline \multirow[t]{3}{*}{4} & PA 255 & 3.54 & -1.12 & -0.006 & 35.87 & 0.41 & -1.24 & 36.00 & -0.69 & -0.56 & 159.33 & 1.52 & -0.82 \\
\hline & Population Mean & 3.70 & & & 37.62 & & & 37.31 & & & 156.90 & & \\
\hline & S.E. $(\mathrm{m}) \pm$ & 0.034 & & & 0.42 & & & 0.34 & & & 0.50 & & \\
\hline
\end{tabular}


Among them PAIG 346 x JLA 794 was widely stable followed by PAIG 346 x DWDa 1402, PA 785 x CNA 449, PAIG 346 x CNA 449, PAIG $346 \times$ HD 514 and PA $801 \times$ HD 514 . These crosses possessed by value greater than unity indicated their high response towards elevated environmental quality for crop growth.Among all the characters, days to maturity was the highly stable character as twelve parents and twenty four crosses found to be highly stable across the environments. It was followed by harvest index and days to 50 $\%$ flowering where ten and nine parents each showed stability across the environments.

The lowest stability was observed for lint index followed by seed cotton yield per plant. In general fibre parameters remain least affected by environment than yield contributing and earliness traits. For remaining characters eight parents showed stability for ginning percentage. Seven parents each observed stable for days to $50 \%$ boll bursting, plant height, number of sympodia per plant and boll weight. Six parents each found stable for number of bolls per plant and seed index. Similar findings were also reported by Chahal et al., (2001), Shashibhushan and Patel (2003), Nirani et al., (2004), Pund and Dev (2006), Khan et al., (2008), Gumber et al., (2009), Deshmukh and Deosarkar (2015) and Balkrishna et al., (2016).

Among the crosses, thirty seven crosses found stable for plant height, twenty seven for boll weight, twenty five crosses for seed index, twenty four each for number of bolls per plant and days to maturity, twenty three each for days to $50 \%$ flowering and number of sympodia per plant, twenty two for seed cotton yield per plant, twenty one for harvest index, nineteen for lint index and eighteen for days to 50\%boll bursting. Among the crosses, PAIG 346 x JLA 794, PAIG 346 x DWDa 1402, PA 785 x CNA 449, PAIG 346 x CNA
449, PAIG $346 \times$ HD 514 and PA $801 \times$ HD 514 were identified as best combinations for stability which have also responded well to favourable environments. Their stable performance was partly attributed to the highly stable nature of their parents in the said environments, stability of the yield contributing traits for which they were observed and their individual buffering capabilities.

The data of the present study revealed that the hybrids had higher mean value for seed cotton yield than the parents. Similarly, regression coefficients for hybrids were superior to parents. Thus, in the present study hybrids exhibited superiority over parents for stability. These results are in agreement with the earlier findings Shashibhushan and Patel (2003), Patel and Patel (2006), Pund and Dev (2006), Khan et al., (2008), Singh et al., (2014), Balkrishna et al., (2016) and Patil et al., (2017).

\section{References}

Balakrishna, B., Chenga Reddy, V. and Lal Ahamed, M. 2016. Stability analysis for seed cotton yield \& its component traits in inter-specific hybrids of cotton $(G$. hirsutum $\times G$. barbadense) Green Farming 7 (5): 1013-1018.

Chahal, G.S., Prakash, R.S., Sohu, R.S. and Nagi, P.S. 2001. Genotype x environment interaction for fibre quality traits in upland cotton (Gossypium hirsutum L.). J. Indian Soc. Cotton Improv., 26(3):134-137.

Deshmukh J. D. and Deosarkar D. B. 2015.Stability analysis in upland cotton (Gossypium hirsutum). Multilogic in Science 4 (3): 155-166.

Gumber R.K., M.S. Gill, J. S. Gill and Dharminder Pathak. 2009. Evaluation of Gossypium arboreum L. genoptypes for genotype environment interaction and 
stabilty of performance. IUPJ. Genetics and Evolution. 24 (1): 164-175.

Khan, N., Naveed G.M. and Khan N.I. 2008. Assessment of some novel upland cotton genotypes for yield constancy and malleability. Int. J. Agri. Biol., 10: $109-111$.

Nirani, K.S., Chhabra, B.S. and YagyaDutt.2004. Genotype x environment interaction for yield and quality traits in GMS based hybrids of G. hirsutum L. J. Cott. Res. \& Dev., 18 (2): $128-131$.

Patel, A.D. and Patel, U.G. 2006. Studies on heterosis, combining ability and stability of performance in upland cotton (G. hirsutum L.). Ph.D. Thesis submitted to N.A.U., Navsari.

Patil, A. E., Deosarkar, D. B. and Kalyankar, S. V. 2017. Impact of genotype $x$ environment interaction on the heterosis and stability for seed cotton yield on heterozygous and homozygous genotypes in cotton. Indian J. Genet.77 (1): $119-125$.

Pund, M.M. and Dev, D.V. 2006. Stability of yield and other quantitative traits in upland cotton. J. Cott. Res \& Dev., 20 (2): 181-184.

Shashibhushan, D. and Patel, U.G. 2003. Genetic architecture of yield and its components of conventional, GMS and CMS based hybrids in American cotton (G. hirsutum L.) Ph.D. Thesis submitted to G.A.U., S.K. Nagar.

Singh S., Singh V.V. and Choudhary A.D. 2014. Genotype x environment interaction and yield stability analysis in multi environment. Tropical and Subtropical Agroecosystems, 17: 477482.

\section{How to cite this article:}

Chinchane, V.N., D.B. Deosarkar and Kalpande, H.V. 2018. Stability Analysis for Seed Cotton Yield and its Component Traits in Hybrids of Desi Cotton (Gossypium arboreum L.). Int.J.Curr.Microbiol.App.Sci. 7(09): 1000-1012. doi: https://doi.org/10.20546/ijcmas.2018.709.119 